\title{
Ética mundial y
cooperación al desarrollo
}

\author{
Joaquín García Roca*
}

El futuro de la cooperación al desarrollo depende, en buena medida, de su capacidad de confrontarse con la ética mundial que se despliega como horizonte de expectativas y posibilidades en función de un mundo único, ${ }^{1}$ como utopíaenergía para la promoción y activación de alternativas en función de

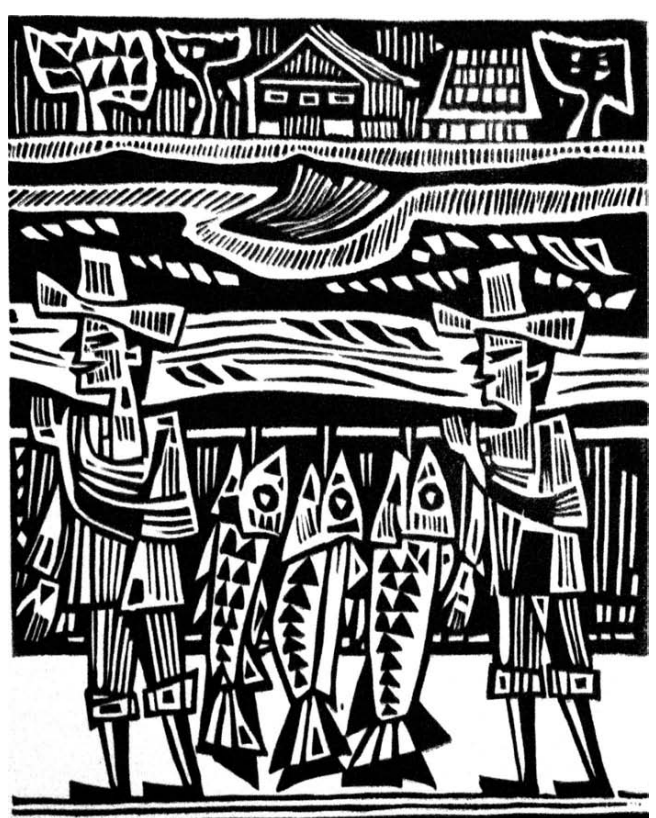

||||||||||||||||||||||||||||||||||||||||||||||||||||||||||||||||

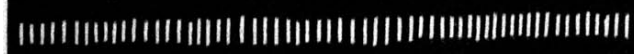

Oficios del río, xilografía a color. 1983 acción y la cultura de la satisfacción, que resultan excluyentes. El horizonte de la cooperación avanza en la construcción de la interdependencia mundial que conforma la geografía actual de lo humano. Asimismo, la ética mundial ofrece provisiones para la cooperación al desarrollo en la medida que activa los potenciales de un nuevo vínculo social y suscita una pluralidad de actores, que se articulan entre sí. Finalmente, la ética mundial es como el freno de emergencia que proponía Walter Benjamín al observar que el progreso producía pirámides de sacrificios. y de este modo desactiva el pragmatismo de la

Datos del autor: Profesor invitado de la Maestría de Gestión del Desarrollo (UPS), Universidad de Valencia y Centro de Estudio sobre Migraciones (CeiM). 
La cooperación para el desarrollo descubre estas tres provisiones éticas desde el lado oscuro de la historia, desde las espaldas del progreso y en permanente connivencia con los excluidos del mismo. Hay lugares, como propuso la sociología crítica y la pedagogía de la liberación, que desvelan el tamaño de la injusticia con la misma nitidez que se conoce una habitación oscura acercándose a la pared. ${ }^{4} \mathrm{Al}$ conocer el sistema desde sus propios límites, la cooperación se inmuniza tanto contra los pesimismos estériles como ante los optimismos ingenuos. "Quien diagnostique hoy un crepúsculo sin amanecer es que está ciego, y quien hable de un amanecer sin crepúsculo es un ingenuo".5

La pedagogía de la liberación propuso pensar desde los últimos y no capitular ante ningún poder; propuso hablar, desde la otra cara del mundo, "desde los últimos de la tierra, desde el Gran Sur".6 Desde esa experiencia ética se puede invocar el sufrimiento de las víctimas para lograr el lugar apropiado, como un día hizo Bartolomé de Las Casas y Antonio Montesinos, quienes en los albores de la modernidad pudieron ver el mundo de los conquistados, de los abatidos, de los excluidos, de los sin poder. $^{7}$

Los retos y oportunidades de una cooperación diferente, crecida, mejor, negociada, con relaciones de reciprocidad y solidaridad es capaz de recuperar su valor ético desde los procesos que están encapsulados en la realidad misma, como los brotes de invierno.

Para abordar estas tareas se impone recrear el principio de responsabilidad ${ }^{8}$ en función de la supervivencia del planeta y las posibilidades de una vida digna para las generaciones futuras. El principio de responsabilidad significa apremio para la decisión, así como pluralización de los actores sobre un trasfondo de incertidumbres.

¿Qué procesos hacen posible la cooperación al desarrollo? ¿Cómo se puede construir un nuevo vínculo mundial? ¿Qué fuerzas sociales la sostienen? ¿Qué modo de política puede encaminar este modo de cooperar?

\section{La producción ética de la interdependencia social}

Un proceso social, que se convierte en generador ético de cooperación, es el nacimiento de la interdependencia planetaria, cuyos sismógrafos son la conciencia del destino común de la tierra, la triple globalización que convierte el mundo en único y desigual, el terrorismo internacional que ha des-localizado los peligros y, finalmente, los procesos migratorios, que desplazan a 180 millones de personas de sus lugares de residencia.

\section{Los sismógrafos de la interdependencia}

La conciencia ecológica anuncia el nacimiento de un mundo interdependiente; en él se muestra la unidad de los seres humanos con la tierra; formamos parte de un organismo vivo, inacabado y en proceso; no somos señores de la tierra, acaso simples responsables de la misma; no somos el centro de la tierra sino que dependemos de ella. Los seres humanos somos tierra que ama, espera, desea, ora. El grito ecológico incorpora hoy a los pobres que son los seres vivos más amenazados de la tierra. ${ }^{9}$

Por su parte, la globalización hace que las mercancías lleguen selectivamente a todos los rincones del planeta, en los que se encuentran ya los tres sacramentales de la globalización: la coca-cola y/o el Mcdonal, la maquila que interconecta el producto que se ultimará en Taiwan y la tarjeta de crédito, que globaliza las finanzas. La globalización puede tener como horizonte "la globalización de la nada" o el nacimiento de un mundo único e interconectado por la vida. ${ }^{10}$

Asimismo, el terrorismo internacional visibiliza la pérdida de domicilio de los peligros, sin morada, ni territorio, ni frontera, ni clase social. Se le busca en Irak y está en las puertas del Pentágono; se le escudriña en forma de arsenal atómico y está en forma de humillación. A la luz del terrorismo internacional que la interdependencia es el destino del que depende la superviven- 
cia de los humanos. El terrorismo tiene suficiente con el odio que no tiene nación, ni clase, ni religión, sino que se domicilia en todos los entresijos de la realidad. Para afrontar estas amenazas, las instituciones locales y los estados nacionales se muestran insuficientes "nunca fueron concebidos como instrumentos para abordar los peligros y las amenazas globales"11 (Rifkin: 344).

Finalmente, las migraciones visibilizan, por su parte, la movilidad social y la imposibilidad de las fronteras ante el fenómeno estructural de la desigualdad. La desigualdad es el efecto llamada, capaz de empujar a unos a morir en el umbral de Europa, en el estrecho de Gibraltar o en cualquier río Grande, intentando alcanzar un paraíso parido por las ondas de televisión. "Nadie pondrá limites, en palabras de Bachir, al hambre de dignidad y a los deseos de expectativas de futuro. Y si morimos en el estrecho es que Alá lo ha querido así”. Los inmigrantes son los voceros de la interdependencia. Ellos denuncian la gran contradicción de querer interconectar el mundo sólo con las mercancías, los productos o las finanzas y excluir a las personas. ${ }^{12}$

\section{Los gérmenes de la interdependencia}

En la actualidad, está muriendo un proyecto de civilización que se construyó en torno al valor de la independencia e impregnó los mejores esfuerzos del siglo XX, en todos los ámbitos de la realidad.

En el espacio político, la autonomía e independencia marcaron la brújula para la construcción de una nación libre como ideal de los pueblos. En tiempos de colonialismos, fue la condición de la libertad y del desarrollo, y seguirá siéndolo para aquellas naciones sometidas a la tiranía interna y al imperialismo externo. Después de lograr la independencia de las naciones a lo largo del siglo XX, nos adentramos ahora en la construcción de la interdependencia mediante alianzas regionales y creación de unidades políticas más amplias que las nacionales. Hoy nace otro horizonte, que disuelve las fronteras, el den- tro y el fuera, lo propio y lo ajeno; las fronteras ya no funcionan para producir la identidad y proteger a los ciudadanos. "No se puede basar la seguridad en los muros", y "la soberanía es una quimera", como recordaba recientemente el analista norteamericano Benjamín Barber. ${ }^{13}$

En el ámbito educativo, la finalidad de la educación se orientó a la creación de sujetos autónomos, que puedan valerse por sí mismos; el valor educativo esencial era la creación de personas autónomas y autosuficientes. Recientemente, el Informe DELORS ampliaba las finalidades educativas mediante el "aprender a convivir y aprender a ser" en contextos de mundialización. Tan importante es el aprendizaje de conocimientos y habilidades, como adiestrarse en el arte de vivir juntos. ${ }^{14}$

\section{La cooperación en tiempos de mundialización}

Si en tiempos de independencia fuimos capaces de crear instituciones que las gobernaran, ahora procede hacer lo mismo con la interdependencia. De hecho se está construyendo a través de un proceso, a la vez económico, político y cultural, que crea un mundo único e interconectado por los intereses, que mueven a los capitales financieros a buscar beneficios económicos en todos los lugares del planeta; por los problemas que requieren instituciones políticas para gobernar los asuntos comunes que afectan a toda la humanidad y por las causas que favorecen la creación de otro mundo mejor y posible como horizonte moral de la humanidad. La confluencia de intereses, problemas y causas hace que la globalización no sólo produzca un mundo único sino también desigual y antagónico. ${ }^{15}$

La difícil coexistencia entre un ideal, que postula un mundo único (mundialización), un sistema económico, que tiene la desigualdad como motor de su desarrollo (globalización de los intereses) y un proyecto político que postula instituciones mundiales (globalización de los pro- 
blemas), hace particularmente densa la cooperación al desarrollo.

\section{La globalización de los intereses}

La globalización más visible y exitosa es la económica, que es hoy el vehículo a través del cual se ha formado un capital mundial mediante la eliminación de todas las barreras para la circulación de bienes y de servicios. En las cuestiones de producción, distribución y comercialización, cada vez tiene menos sentido hablar de enclaves territoriales o de Estados nacionales, en su lugar se asiste al flujo de mercancías y capitales para traspasar las fronteras; hay un poder económico,

Para la globalización neoliberal, la cooperación al desarrollo es contraproducente porque rompe las leyes del mercado; es ineficaz porque no logra lo que se propone, como es acabar con la pobreza; y es insignificante porque deja las cosas como estaban.

que avanza como el conquistador y lleva inscrita una dinámica depredadora, que va dejando por el camino a todos los retardatorios.

Cuando los intereses económicos se constituyen en el motor de la globalización, ésta propone la liberalización y las privatizaciones por encima del bien común, reduce el gasto social y los presupuestos dedicados a los bienes públicos, restringe la disciplina fiscal y los impuestos, favorece la libertad de movimientos de capitales y reduce el movimiento de los trabajadores. Esta globalización neoliberal ha institucionalizado el poder de los mecanismos económicos -mercados y empresas- por encima de los derechos humanos, de los proyectos políticos, de las necesidades sociales y medioambientales. ${ }^{16}$

Ciertamente, esta globalización económica ha ampliado la oferta de bienes y servicios disponibles por todo el mundo, pero también ha pro- ducido un desfase moral en el que 1.200 millones de personas viven con menos de un dólar diario y el $20 \%$ de la población mundial disfruta del $80 \%$ de su renta. Las inversiones especulativas privan sobre las productivas: la mayor parte de los seis mil millones de dólares que circulan diariamente en los mercados globales son capitales especulativos. El resultado es un mundo más comunicado, pero más desigual y asimétrico.

Algunas consecuencias golpean fuertemente la visión cooperante: el sometimiento de la política a la economía, con la reducción de los espacios para la política; el aumento de las desigualdades sociales, con brechas crecientes entre el Norte y el Sur y dentro de cada uno de ellos; y las crisis ambientales que modifican las condiciones de vida. Pero sobre todo, importa advertir las consecuencias antropológicas que están por debajo de este proyecto globalizador, que convierte al hombre en un depredador, competitivo y devorador.

Para la globalización neoliberal, la cooperación al desarrollo es contraproducente porque rompe las leyes del mercado, es ineficaz porque no logra lo que se propone, como es acabar con la pobreza, y es insignificante porque deja las cosas como estaban. ${ }^{17}$

\section{La globalización de los problemas}

Junto a esta globalización, asistimos igualmente a la necesidad de afrontar conjuntamente los problemas; los riesgos y las amenazas no tienen domicilio sino que andan por todos los pliegues de la realidad; no son calculables ni previsibles desde un único territorio, ni por unos individuos, ni por unos estados, sino que caracterizan el actual modo de vida. Lo que sucede dentro del globo, atañe a todos y afecta a cada uno.

La emergencia de estos problemas globales ha producido otra dimensión de la globalización que consiste en la creación de instituciones mundiales. Gracias a este proceso, se tutelan valores comunes sobre los derechos humanos, sobre el medio ambiente, sobre la mujer, sobre el desa- 
rrollo sostenible, sobre la alimentación, sobre el agua... Las Cumbres Mundiales son los sismógrafos de este proyecto de globalización basado en la conciencia de los peligros.

Las instituciones locales y nacionales se muestran incapaces de gobernar los nuevos problemas que trascienden las fronteras $y$, de este modo, se postulan

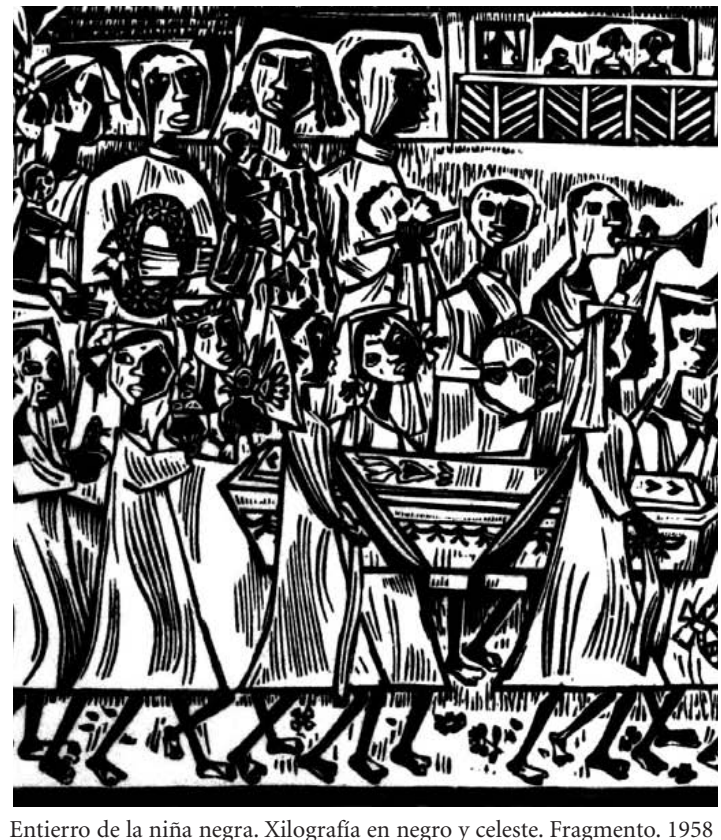
nuevas formas de de-

recho internacional y de estructuras de "gobernanza". Si las amenazas son globales, el remedio no puede ser nacional, aunque haya que tomar medidas en este plano. Frente a sus amenazas, es pertinente una alianza entre todos los estados, las instituciones locales e internacionales, los distintos actores, cuya última expresión es la Declaración del Milenio.

Tradicionalmente, las guerras clásicas eran protagonizadas por ejércitos permanentes, que actuaban de forma autónoma y se situaban en un espacio y tiempo bien definidos. Los peligros, por el contrario, estallan de forma capilar y disuelta, desde abajo como procesos endógenos, que penetran en todos los poros de la vida social en formas imprevisibles. Se producen en cualquier lugar, en cualquier calle, en el metro; basta con que alguien prefiera otro club de fútbol, que vista de otro modo, que hable otra lengua, que precise de una silla de ruedas o que se toque la cabeza con un pañuelo. ${ }^{18}$

Para la globalización de las responsabilidades, se propone la creación de instituciones internacionales, basadas en la posibilidad de alianzas y vinculaciones internacionales.

Con frecuencia, la construcción de esta segunda globalización no sólo prescinde de la par- ticipación ciudadana sino que soslaya los derechos humanos. Lo ha dicho inequívocamente Mary Robinson, Alta Comisionada de la ONU para los Derechos Humanos, "el orden y la seguridad nacionales han significado con frecuencia una restricción de la democracia y de los derechos humanos". ${ }^{19}$ Lo advertía con preocupación el Secretario General de Naciones Unidas ante el Consejo de Seguridad el 28 de enero de 2002: "Todos deberíamos tener claro que no hay ninguna contradicción entre una acción eficaz contra el terrorismo y la protección de los Derechos Humanos. Por el contrario, creo que, a la larga, comprenderemos que los derechos humanos, junto con la democracia y la justicia social, constituyen la mejor profilaxis contra el terrorismo. Aunque está claro que son necesarias la vigilancia para prevenir los atentados terroristas y la firmeza a la hora de condenarlos y castigarlos, sería contraproducente sacrificar en el proceso otras prioridades clave como los derechos humanos". El director del Centro Carr de Política sobre Derechos Humanos de Harvard, M. Ignatieff, afirmaba que "el problema es saber si, tras el 11 de septiembre, la era de los derechos humanos ha llegado a su fin".

Esta segunda globalización de los problemas se sustancia últimamente en torno a las emergencias. En los últimos años, cooperar significa mayoritariamente atender a situaciones de emergencia originadas por la guerra, las carestías, las hambrunas, las catástrofes naturales a través del amplio abanico de ayudas humanitarias. En la actualidad, rondan cerca de 40.000 organizaciones no gubernamentales de carácter internacio- 
nal, que gestionan el $20 \%$ de toda la ayuda internacional a los países empobrecidos.

Sin negar su papel decisivo, es evidente que este desplazamiento está produciendo efectos perversos, encubrimientos y retóricas que dificultan la cooperación al desarrollo.

Las emergencias, que requieren ayuda humanitaria, desplazan a las situaciones de subdesarrollo, que demandan cooperación al desarrollo. Con frecuencia, las Organizaciones no gubernamentales para el desarrollo (ONGDs) han sido utilizadas como ambulancias para reducir, controlar o asistir tanto las emergencias como las injusticias. El primer efecto de esta identificación ha sido el debilitamiento de la cooperación, que muestra una gran fatiga, quizá porque los resultados son lentos y poco vistosos en los cortos períodos electorales; la emergencia, por el contrario, goza de alta estima social y política. ${ }^{20}$

El segundo efecto de la identificación de ayuda humanitaria y cooperación ha sido la creación de grandes agencias humanitarias que en nombre de la eficacia y la eficiencia se han constituido en auténticas empresas de servicio. Tan alarmante es esta progresiva burocratización que la revista de los combonianos Mundo negro ha llegado a escribir que "El alma propia de las ayudas esta cambiando: la valencia solidaridad es siempre más débil, la valencia auto-reproducción de la agencia humanitaria es siempre más potente". 21

En tercer lugar, parece inevitable que las organizaciones humanitarias sean instrumentalizadas y utilizadas en el contexto de la guerra. Hay una cooptación de las organizaciones de cooperación que crea una dependencia material y cotidiana de las estructuras militares". La contraposición entre las ONGDs y el ejército pertenece a la historia", decía un militar en Kosovo. Al convertirse en ambulancias mundiales, que socorren a los bombardeados en nombre de los que bombardean, sirven para darle un barniz humanitario a las guerras, lo que Chomky ha llamado humanitarismo militar; se trata de crear un aparato ideológico y material para socorrer a las victimas con el fin de aumentar el consenso interna- cional a la operación militar. El lanzamiento de paquetes humanitarios junto con las bombas, ocurrido en la guerra de Afganistán, constituye la mejor escenografía de esta realidad. ${ }^{22}$

Las propias organizaciones de cooperación sufren la colonización, por parte de las emergencias, cuyas intervenciones son muy volátiles y dependen mayormente de la captación de donaciones. Impone un estilo a los proyectos de intervención que recupera el paternalismo, ya que son preparados en un despacho, sin conocer el contexto local. En tiempos de emergencia parece que lo importante es la intervención rápida con la misma metodología, la misma estrategia, los mismos dispositivos. En las situaciones de emergencia está argumentada la intervención jerárquica, sin consulta ni participación. Incluso está justificada la intromisión en los asuntos internos del país en nombre de la seguridad, cuando realmente es una simple injerencia.

La emergencia, al contrario que la cooperación, carece de conciencia crítica, abandona los procesos sostenidos por la acción puntual a corto plazo y desprecia las capacidades locales. Reduce a los pueblos a simples comparsas y a los nativos a meros empleados de sus burocracias. Están pocos meses, viajan en carros especiales, frecuentan restaurantes para extranjeros, se reúnen entre ellos; corren de emergencia en emergencia sin un proyecto ni una política de trasformación. Finalmente, el encubrimiento mayor, sin embargo, es aquel que en nombre de la emergencia humanitaria convierte el sufrimiento en espectáculo.

\section{La mundialización de la solidaridad}

Hay una tercera globalización, que mundializa lo humano y las causas solidarias, a través de la cooperación. Por la mundialización de la solidaridad, todos los seres forman una realidad orgánica e interconectada; la conciencia humana se amplía a escala planetaria y se ha creado una nueva alianza del ser humano con la naturaleza. Se trata no 
sólo de un cambio de escala, que pasa del Estadonación al sistema-mundo, sino de uno de residencia mental y cordial. Un mundo único y solidario ha impregnado todos los sueños diurnos elaborados tanto por las tradiciones religiosas, que lo hacen derivar de las manos divinas, como por la cultura laica, que lo sitúa en el interior de un proyecto colectivo de dignidad y de justicia.

Si la primera globalización está impulsada por las fuerzas económicas y por las empresas multinacionales, la segunda por instituciones políticas nacionales e internacionales, la cooperación al desarrollo vehicula la tercera, que se alimenta de valores y de sueños de gentes y pueblos, que imaginan otro mundo posible y necesario. El paso de la primera globalización económica que convierte el mundo en un supermercado, a la segunda globalización que despoja a los Estados nacionales de su autosuficiencia requieren de la tercera globalización como respuesta a los procesos globales de conquista, colonización y empobrecimiento.

\section{En las situaciones de emergencia es-} tá argumentada la intervención jerárquica, sin consulta ni participación. Incluso está justificada la intromisión en los asuntos internos del país en nombre de la seguridad, cuando realmente es una simple injerencia.

Los portadores de esta mundialización son la nueva geografía de la cooperación al desarrollo que "comprende una multitud de luchas locales, que promueven a nivel transnacional una democracia sustancial como contrapeso al neoliberalismo". ${ }^{23}$ Los grandes sismógrafos son los movimientos sociales: los movimientos de mujeres que se sacuden el yugo del patriarcalismo milenario y buscan definir su identidad en reciprocidad con lo masculino; los movimientos religiosos, que propugnan un diálogo de religiones más allá de sus respectivas ortodoxias; los movimien- tos a favor de la tierra, que proclaman el destino universal del planeta, la defensa de los derechos humanos que gritan la común dignidad desde todos los rincones del mundo; los movimientos de resistencia antiglobalización, que se comprometen con la paz, los derechos humanos, la defensa de la infancia, el desarrollo, el comercio justo, la condonación de la deuda del Tercer Mundo, el medio ambiente o el género. La economía social que rompe a pequeña escala las leyes del capitalismo salvaje y depredador y de esta forma testifica que la lanzadera de David frente a Goliat pertenece a la tradición popular. Las organizaciones de voluntariado que canalizan la acción de ciudadanos en organizaciones solidarias y se transforman en personas activas a través de las prácticas del don y de las relaciones de ayuda. Las tres constituyen hoy la fisonomía de la cooperación al desarrollo.

Adquiere actualidad la advertencia que hizo en su día el premio Nobel de Literatura, el bengalí Tagore, "Durante más de un siglo hemos sido arrastrados por el próspero Occidente detrás de su carro, ahogados por el polvo, ensordecidos por el ruido, humillados por nuestra propia falta de medios y abrumados por la velocidad. Accedimos a admitir que la marcha de este carro era el progreso, y que el progreso era la civilización. Si alguna vez nos aventurábamos a preguntar "progreso hacia qué y progreso para quién", se consideraba que abrigar ese tipo de dudas acerca del carácter absoluto del progreso era una rasgo excéntrico y ridículamente oriental. Recientemente, hemos comenzado a percibir una voz que nos advierte que hemos de tener en cuenta no sólo la perfección científica del carro, sino la profundidad de las fosas que surcan su camino".

\section{La producción ética del víncu- lo mundial}

La refundación ética de la cooperación necesita recrear un nuevo vínculo mundial, que sirva de cemento y fundamento de todo el edifi- 
cio. Cada formación histórica ha sido posible mediante un nuevo pacto social. Piénsese, por ejemplo, en el nacimiento del Estado de bienestar, fue posible porque hubo un pacto entre los enfermos y los sanos, los trabajadores y los parados, los jóvenes y los ancianos... Era el vínculo necesario para afrontar los efectos de la Segunda Guerra Mundial. La Unión Europea está siendo posible gracias al pacto entre los Estados de un determinado territorio, que se sustancia en la constitución europea.

\section{Un vínculo mundial}

En la actualidad, se vislumbra la puesta en marcha, a escala planetaria, de un nuevo vínculo social. Hay muchos intentos por reformularla: la pertenencia a la misma familia humana, el destino de la tierra, la unidad de la especie, los derechos humanos, la ciudadanía cosmopolita.

Los seres humanos hemos ensayado históricamente todos los mecanismos para construir un dentro y un fuera, para establecer un nosotros y un ello. Se han creado cientos de oposiciones para definir la pertenencia y la identidad: la edad, la clase, el grupo étnico, la comunidad, la religión.

Los elementos étnicos sirvieron en un principio para marcar las diferencias y señalar fronteras; de este modo, se declaraban importantes las propiedades personales adquiridas por descendencia, las características físicas reconocibles por el aspecto, asociadas a propiedades naturales.

Los elementos religiosos desplazaron a los étnicos. Los grupos humanos se conforman como comunidades en torno a creencias y convicciones que dirimen las cuestiones del origen, el destino y ofrecen normas para vivir y morir con sentido. La potencia de lo sagrado cimentaba al grupo, hasta el punto que "si los dioses no cambian, nada ha cambiado".

Los elementos culturales ampliaron la versión religiosa. El nosotros estaba cohesionado por la herencia colectiva, por las ideas, costumbres y prácticas que configuran la vida. "Nosotros hace- mos esto, que es bueno; y ellos hacen lo otro, que es malo". La cultura del grupo se representaba como una reproductora de copias idénticas. Muy pronto se entendió que la cultura no se asemeja tanto a una fotocopiadora, cuanto a un concierto improvisado.

Con la primera modernidad, la identidad del nosotros se construye con elementos políticos. El nacimiento del Estado-nación se convierte en referente para marcar fronteras: el pasaporte o el documento de identidad confieren estatus nacional y consecuentemente distribuye la ciudadanía. La modernidad entendió que la justicia, la igualdad, los derechos... han de aplicarse en el marco jurídico de un Estado.

\section{Lo universalizable}

La cooperación no acepta los criterios étnicos, religiosos, culturales o políticos como fundamento de la nueva ciudadanía cosmopolita; y en su lugar reconoce un vínculo más radical: la responsabilidad común ante el sufrimiento humano. Una responsabilidad que no tiene límites territoriales, ni étnicos, ni religiosos, ni políticos, ni ideológicos sino que asume como suyo el sufrimiento de los perdedores, de los empobrecidos. Sólo la reacción ante el sufrimiento injusto alcanza un horizonte universal, que se despliega en resistencia unas veces y en indignación otras contra las causas del sufrimiento injusto.

La pregunta ética decisiva hoy es saber si hay algo que pueda ser universal; unos han visto lo universalizable en la dignidad, cuyo nombre actual son los Derechos Humanos, que conforman el consenso mínimo alcanzado para fundamentar un nuevo consenso mundial. Otros han visto en la pertenencia a la familia humana o al género humano, el elemento fundamental de la universalización.

Recientemente, se ha recuperado la vieja idea hobbesiana para fundamentar el vínculo mundial. En el contexto de los atentados terroristas, el miedo y la inseguridad frente al Otro se convierten en la instancia última. Estamos uni- 
dos porque necesitamos defendernos. "Porque el terrorismo afecta a todos, todos debemos unirnos ante él". El miedo común y el peligro compartido es lo máximo universalizable, que fundamenta la cooperación al desarrollo, en la medida que el hambre es un dominio de inseguridad. Se explica de este modo que el $50 \%$ de los presupuestos estatales dedicados a la cooperación, se usen en la reconstrucción de Irak. El miedo crea más responsabilidades que la justicia.

Cuando el otro es portador de amenaza, es la defensa ante él la que justifica la colaboración entre los agredidos. Cuando el emigrante económico es un enemigo, que quita trabajo, trae delincuencia y produce inseguridad, el vínculo social será el miedo a perder lo que uno cree que le pertenece. El miedo y la seguridad producen una vinculación basada en mecanismos de defensa. Las consecuencias son inevitables: se exige que los otros sean objeto de controles especiales. Lo que produce un ambiente de odio contra grupos étnicos, que en última instancia cohesiona a una cierta sociedad, y de este modo, se ha recrudecido la necia imaginería maniquea del bien contra el mal. El miedo distorsiona las agendas de los gobiernos y desplaza la cooperación hacia su propia seguridad, y las energías sociales se focalizan hacia la preocupación por la seguridad, real o ficticia.

\section{Responsabilidad común ante el sufrimiento humano}

Podemos y debemos recrear el universalismo moral sobre otras bases, que actúen con autoridad incondicional y pretensión de verdad. Como advirtió Theodoro Adorno, "dejar hablar al dolor es la condición de toda verdad" y percibir el sufrimiento del otro es la condición indispensable para toda pretensión moral universal, aquello que puede fundamentar las opciones pro-cooperación.

¿Qué puede significar hoy esta responsabilidad universal ante el sufrimiento provocado por la injusticia?
Significa, en primer lugar, que se alcanza la universalidad a partir de una parcialidad, de los últimos, de los que están fuera, de los que ven negado su ser y sus derechos (E. Dussel) Se puede y se debe universalizar desde los débiles, los perdedores, los que no pueden dar la vida por supuesto. Hay un sufrimiento en la realidad, que pertenece a todos, tanto si se produce en Lima como en Bagdad. Cuando los últimos tienen reconocidos sus derechos, los tenemos todos. El lugar de la universalización son las victimas y los perdedores.

Esta memoria del sufrimiento del otro es la base ética de la cooperación. De este modo, la solidaridad se asienta sobre la autoridad de la silla vacía; es una autoridad que se impone absolutamente. El que no está sentado en la mesa tiene la clave y la autoridad del tiempo. Como afirma Agnes Heller "la silla vacía espera al Mesías y mientras la silla esté ahí, emite bramidos y admoniciones, incluso patéticos, para que se le tenga en cuenta. Todo el resto es pragmatismo". La cuestión hoy no es saber quien ocupará la silla vacía, sino saber si la política democrática moderna se realizará bajo la constelación de la silla vacía, o si en nombre de la modernidad se deberá renunciar a esta prioridad. ${ }^{24}$

Cuando la centralidad de la gestión política gira en torno a los intereses de la sociedad de la abundancia y, dentro de ella, en torno a los de las clases medias, desaparece la cooperación. Esta centralidad, que escucha el hambre y la sed de justicia, es la categoría central en la fundación ética de la cooperación.

Si logramos poner en el centro a los últimos, la cooperación será un camino de justicia con los que están peor situados.

\section{Dar y recibir}

En el interior de la cooperación bulle un doble potencial, que se sustancia en el dinamismo del dar y en el dinamismo del recibir. La cooperación es viable en la confluencia del dar y del recibir, de ayudar y ser ayudados, de cuidar y ser cuidados, de educar y ser educados. Con fre- 
cuencia, los dinamismos se contraponen y son mutuamente excluyentes de modo que unos dan $y$ otros reciben, unos cuidan y otros son cuidados, unos educan y otros son educados. La cooperación, desde esta perspectiva, no es una ayuda de los que tienen para los que no tienen. Este tipo de ayuda sólo es un ejercicio del poder.

Cooperar consiste en hermanar el dar y el recibir. La relación de ayuda se siente libre y espontánea, humilde y agradecida a un mismo tiempo. Lejos del ejercicio de dominación y del complejo de quien no tiene nada que recibir se domicilia en la apertura permanente hacia los demás. Como señalaba Hélder Cámara "Nadie es tan rico que no pueda recibir algo ni tan pobre que no pueda dar algo".

La raíz de la solidaridad consiste en dar aquello que hemos recibido, y en recibir aquello que los otros nos dan. De este modo, reconoce el momento de verdad que comporta el dar y el recibir, pero se resiste a fragmentarlos. $Y$ de este modo se alumbra el dominio de la solidaridad. No es manteniendo el mito del capitán del barco como sobrevive la embarcación, sino insertándose en el seno de un movimiento que se sustenta sobre la colaboración y sobre la alianza.

La solidaridad ante el sufrimiento reivindica el dinamismo del dar y recibir como un proceso interactivo entre personas y pueblos, que permite cuestionar la civilización del cowboy, que como afirma Fátima Mernisi, se construye sobre el otro como peligroso, se defiende con las pistolas y crea ranchos para la autodefensa. Esta ideología del cowboy ha enfatizado el concepto de identidad como sinónimo de pureza incontaminada.

La solidaridad como dar-recibir es el viático para salir de las visiones uniformes, de las pretensiones totalitarias y de las ideologías hegemóni- cas, que con frecuencia acompañan las políticas pro-cooperación.

\section{La construcción ética de los actores de la cooperación}

La geografía social de la cooperación anda a la búsqueda de una arquitectura, que urbanice sus desempeños, reconozca el papel de los nuevos actores y sea capaz de articular las sinergias. No todo vale en la cooperación al desarrollo aunque todo el mundo tiene un papel en la cooperación. Sin embargo, la situación actual de la cooperación al desarrollo es caótica a causa del desbordamiento de los actores tradicionales de la cooperación, con la consiguiente descoordinación en sus funciones y tareas. Las Universidades construyen molinos para el regadío en el Sur, mientras los Ayuntamientos crean escuelas para

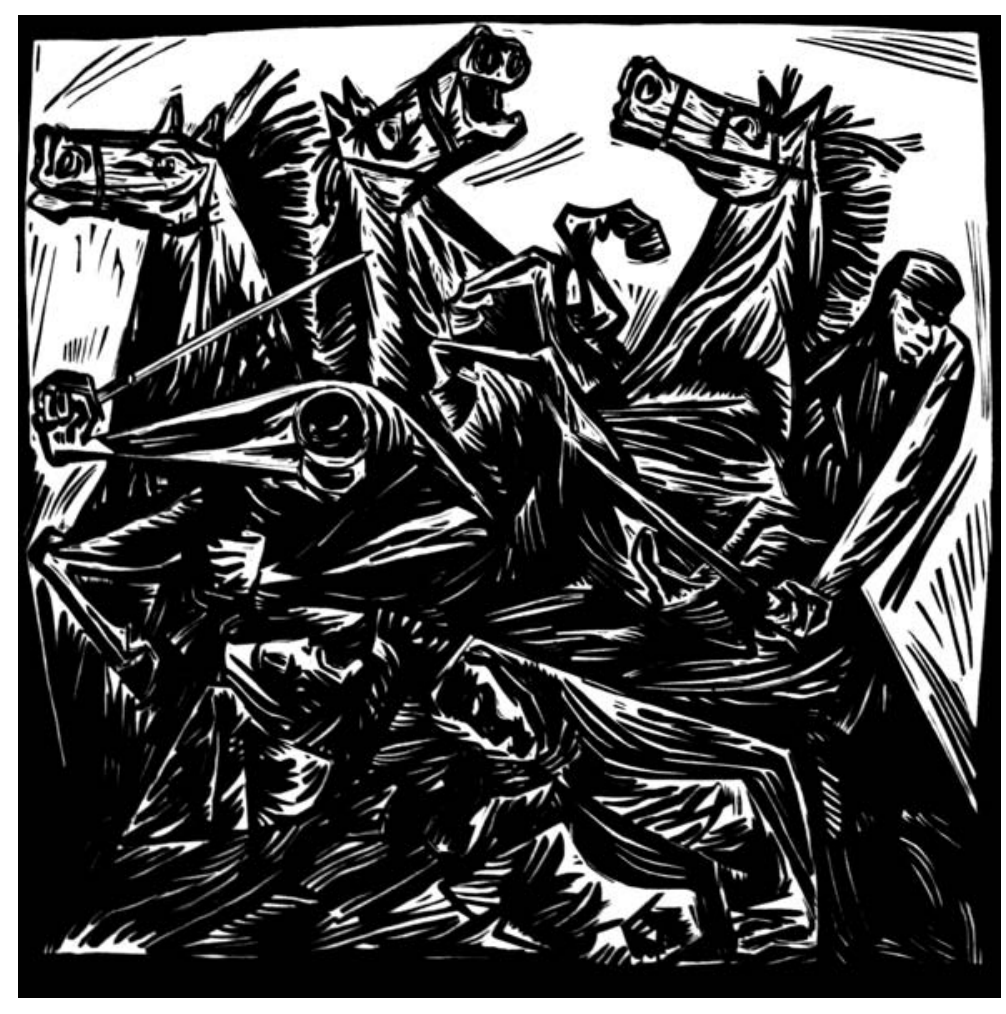

Las caballadas. 1950 
formar cooperantes en el Norte. Las ONG para el desarrollo solicitan subvenciones de los presupuestos públicos, mientras las Administraciones Públicas abren cuentas bancarias para recaudar dinero de los ciudadanos. Los empresarios hacen campañas navideñas para hacer felices a los niños del Tercer Mundo, a la par las iglesias construyen barbacoas para promover viviendas. Mientras los bancos promueven centros sociales en los países en desarrollo y las cooperativas desarrollan microcréditos. ¡El mundo al revés!

\section{Distinguir para unir}

Para urbanizar la cooperación, es necesario distinguir para unir y sólo de este modo se activan reciprocidades entre los actores que interactúan en el mundo de la cooperación. Tan importante resulta la pluralización de los actores de la cooperación como articularles coordinadamente.

\section{El arte de la diferenciación}

La cooperación necesita hoy someterse al principio de diversificación y al arte de la separación, que se despliega en pluralidad de actores (WALZER). Diferenciar los territorios, delimitar las esferas y acotar las competencias es el ejercicio cultural que permitió abandonar la Edad Media. "Por favor...déjennos hacer tranquilos nuestra Edad Media..." le dice Bolívar a un médico francés en la obra de García Márquez El general en su laberinto.

Practicar el arte de la diferenciación es urgente en el ámbito de la cooperación, ya que con él se producen los límites y los dominios específicos. La cooperación debe diferenciar la esfera social y la estatal; en la primera nacen las ONGD y en la segunda las agencias oficiales. Las primeras expresan el dinamismo cooperante de la Sociedad civil; las segundas instrumentan la Ayuda Oficial al desarrollo y los créditos. Las ONGD para el desarrollo despliegan el ca- pital social que anida en la gente; las Administraciones, por su parte, gestionan los presupuestos públicos y crean el marco jurídico. La esfera política debe regular los mercados, los intercambios y las relaciones entre los gobiernos. La esfera social está interesada en crear la conciencia mundial, que se sustancia en la relación entre los pueblos y sus gentes. Si lo primero crea el funcionario de la cooperación, lo segundo crea el cooperante.

La cooperación debe, asimismo, diferenciar la esfera política y la esfera religiosa. La primera debe someterse a criterios de legitimidad política a través de mecanismos democráticos; la segunda se somete a la legitimidad social por la pertenencia libre y la lealtad personal. Si lo primero crea al funcionario de la cooperación internacional; lo segundo, el misionero.

En consecuencia, la cooperación al desarrollo debería diferenciar más de lo que está dispuesto a hacer actualmente entre lo que corresponde a los Estados a través de sus Administraciones y lo que corresponde a la sociedad civil desde sus organizaciones sociales.

\section{El arte de la integración}

El ejercicio de la diferenciación, con ser absolutamente necesario, produce efectos contraproducentes si no se acompaña del arte de la integración. La persistente apelación a los planes integrales y a las estrategias a largo plazo, que se observa en el ámbito de la cooperación, puede verse como un intento de armonizar los actores, comunicar los ámbitos y vincular la economía, la política y la ética; la política con los valores; lo local con lo mundial; lo administrado con lo comunitario; las ONGD y los Estados.

El futuro de la cooperación dependerá en gran medida de que sea capaz de lograr la armonización interna de todos los actores, una vez conquistada la autonomía propia de cada uno de ellos. Resultará decisivo articular las distintas esferas así como los nexos y vínculos necesarios entre las políticas nacionales y las internaciona- 
les, entre el crecimiento económico y el desarrollo humano, entre los cooperantes y los misioneros, entre las administraciones y las ONGD.

Un mayor grado de pluralización de los actores de la cooperación, sólo tiene sentido si se producen entre ellos relaciones sinérgicas, que evitan el aislamiento al tiempo que incrementan la coordinación y el enriquecimiento recíproco. Sinergia es sinónimo de constante cooperación, de interacción empática y funcional de los factores y actores implicados en la resolución de los problemas. Se trata de combinarlos de manera que su actuación acreciente la aportación específica y las fortalezas de cada una de las partes. Recrear la interdependencia a través de prácticas integrales es la tarea pendiente en el ámbito de la cooperación.

\section{La mentalidad suma cero}

¿Cómo distinguir las competencias y los actores de la cooperación? ¿Se puede lograr la articulación y las relaciones sinérgicas entre las distintas esferas? ¿Pueden ser complementarias las distintas lógicas de la cooperación?

La mentalidad de la suma cero impone su ley en ciertos modos de entender la cooperación. Planteados como antagónicos y excluyentes los distintos actores de la cooperación, parece inevitable que cada uno crezca a costa del otro, cristalicen en territorios excluyentes, desarrollen lógicas opuestas y se substancien en prácticas enconadas.

La terca mentalidad de suma cero, postula que para ganar algunos otros tienen que perder; las ganancias del vencedor son matemáticamente iguales a las pérdidas del otro: si en juego hay diez pesos y gano ocho, alguien ha tenido que perder. La imagen tiene una poderosa influencia en la cooperación para el desarrollo. Se acepta resignadamente que "a más políticas de cooperación, menos organizaciones solidarias", o al contrario "a más Sociedad, menos Estado", y no resulta difícil encontrar en las hemerotecas abundantes argumentos que abonan ambas patologías.
El inmenso poder de esta mentalidad expresa la colonización de la cooperación por parte de la racionalidad económica. La cuestión hoy es saber si no hay alguna manera de escapar a la mentalidad de la suma cero en el ejercicio de la cooperación. ¿Podrán coexistir, complementarse o dotarse de apoyos mutuos? La tarea consiste en saber cómo se pueden relacionar distintas esferas de la realidad, al tiempo que se mantienen como realidades diferenciadas. Distinguir para unir, diferenciar para integrar.

En los últimos años existen intentos bien intencionados, que enfatizan el problema de la cooperación en el aumento de los presupuestos destinados al desarrollo. Y en verdad que todo lo que se haga para incrementar los presupuestos será poco ante el alto grado de abandono que tiene la cooperación en las agendas de la hacienda pública; todo lo que se haga para aumentar los presupuestos será poco cuando estamos más lejos que nunca de alcanzar el objetivo fijado por las Naciones Unidas, hace cuatro décadas de que los países ricos destinen al menos el 0,7\% de su Producto Interior Bruto (PIB) a la Ayuda Oficial para el Desarrollo.

Sin embargo, la solución no está en el simple crecimiento presupuestario, por ampliación de lo existente, sin plantearse la racionalidad de los medios. Es una grave patología que las Administraciones agreguen nuevos dispositivos a los ya existentes y sumen nuevos ingredientes a las viejas estructuras, o que las ONGD quieren ser cada vez más fuertes y potentes por ampliación de recursos, de socios y de influencias, sin plantearse la coherencia interna y la racionalidad de las medidas.

Las organizaciones solidarias, también, han sido colonizadas por esta lógica incremental y cada vez más se asemejan a las empresas de servicios, que miden su poder por la cuenta de resultados, por el peso económico y por la fidelización de los usuarios. En este caso, la eficacia sustituye a la participación y la eficiencia a la implicación comunitaria. 
La ilusión acumulativa esconde, pospone y aplaza la imperiosa necesidad de encontrar nuevos modelos de gestión para la cooperación. Se asemeja al espejismo que sufre el maestro cuando quiere vencer el fracaso escolar de su alumno a base de aumentar las horas de clase, en lugar de revisar los métodos de aprendizaje.

\section{Los dinamismos internos de la cooperación}

En el interior de la cooperación coexisten tres niveles perfectamente delimitados y, sin embargo vinculados entre sí. Hay un primer nivel en el cual la cooperación tiene el estatuto de las

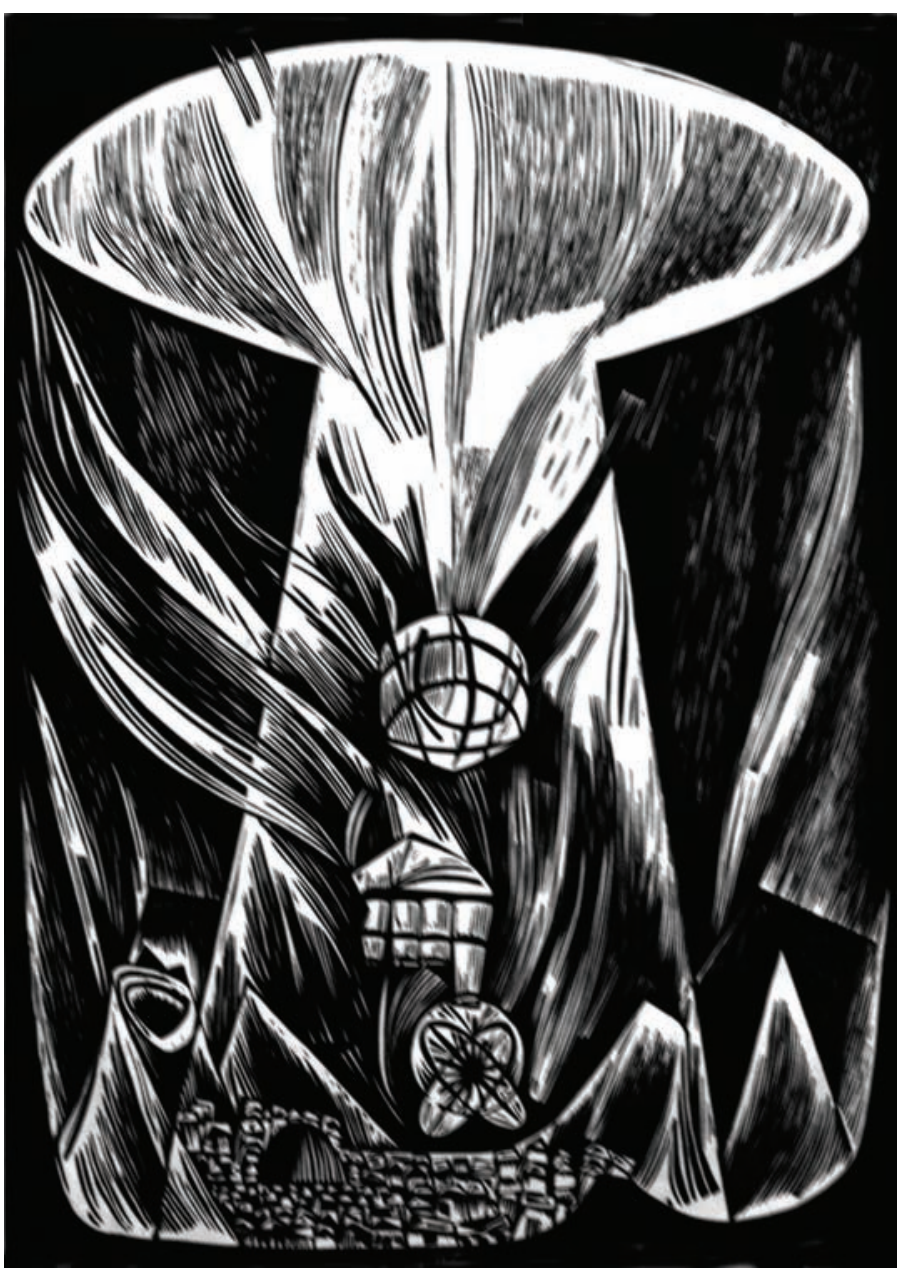

Serie Bomba atómica,1953 virtudes cívicas que se cultivan como hábitos del corazón. En un segundo nivel, la cooperación se despliega como movimiento social con los dispositivos de la presión ciudadana y la acción colectiva. El tercer dinamismo de la cooperación alude a la gestión de proyectos con un alto nivel de institucionalización. Los tres niveles forman un "continuum".

\section{La cooperación como energía cívica}

El primer dinamismo interno de la cooperación se despliega en el cultivo de valores como la solidaridad, la generosidad, la magnanimidad. La cooperación se sustancia en forma de hábitos del corazón o como virtud cívica.

La cooperación es, en primer lugar, un código de conducta que anida en la conciencia y arraiga en el mundo de la vida; se inserta, primariamente, en la experiencia personal y en la vida cotidiana. Este primer nivel constituye la base donde se construye el edifico de la cooperación mediante el sentido de justicia y de solidaridad. Se sustancia en el escenario de los mundos vitales y crea los espacios comunitarios. Se estructura como alianza, se sostiene sobre estrategias cooperativas, tiene su base moral en los sentimientos altruistas. Sin el fortalecimiento de las virtudes cívicas, no hay políticas de cooperación.

Cualquier política de cooperación exige cultivar este nivel, como el árbol necesita alimentar sus raíces. Junto al proceso de institucionalización que vive recientemente el mundo de la cooperación, es necesario activar la responsabilidad personal y colectiva, la solidaridad individual y comunitaria que se expresa en un estilo de vida que pueda universalizarse.

La dignificación de la solidaridad como virtud permitirá dinamizar a la vez el crecimiento en estructuras solidarias y el desarrollo de la responsabilidad personal, articular la garantía jurídica y la iniciativa social, conectar los mecanismos abstractos de protección y la personalización de las relaciones y aunar la gratuidad con la justicia. 
Este nivel de motivaciones y convicciones es más necesario que nunca ya que la actual situación mundial no solicita sólo el ejercicio de la ayuda, sino que requiere la disponibilidad a perder derechos legítimos y cuestionar el propio nivel de bienestar o las prácticas que generan una sociedad patógena para poder superar la brecha de las desigualdades mundiales.

\section{La cooperación como movimiento social}

La cooperación al desarrollo se sustancia, igualmente, en forma de movimiento social, que se sitúa a la mitad del camino entre la virtud privada y el ámbito de la política institucional. Es una acción colectiva que intenta transformar las relaciones sociales entre Norte-Sur, consciente de que el problema del Sur está en el Norte. Este esfuerzo colectivo se activa, se alimenta y se expresa en forma de protesta y movilización social.

Como movimiento social, la cooperación para el desarrollo procura cambios sustanciales en el Norte. Lo decisivo, entonces, es la capacidad de movilizar la sociedad en torno a un desarrollo humano y sostenible, pero sobre todo universalizable; como todo movimiento social, nace en las fracturas de la actual organización mundial; se organiza en función de un desarrollo humano basado en la redistribución de la riqueza, en la promoción de condiciones de vida y en una forma más justa y equitativa de ordenar las relaciones internacionales.

La agenda de la cooperación como movimiento social se centra en torno a la lucha contra la pobreza, la defensa de la tierra, la cultura de la paz, la deuda externa, el comercio justo o las campañas antiminas. En torno a intereses como la defensa y la restauración de formas amenazadas de vida, la inmigración o la supervivencia de la humanidad, se desencadenan en una acción colectiva, cada vez más potente y organizada.

La función principal de las ONGD, en este momento, consiste en promover y activar un te- jido social con capacidad de movilizarse y hacer oir su voz crítica ante las injusticias y los desequilibrios que genera la actual desorganización social. Lo cual no será posible si se convierten en estructuras jurídicas enfrentadas únicamente a gestionar proyectos. La existencia de presupuestos públicos a disposición de las ONGDs mediante subvenciones públicas ha provocado el abandono de la condición de movimiento social.

\section{La cooperación como gestión}

La cooperación como virtud y como movimiento social se sustancia en dispositivos y estructuras de servicio, que gestionan proyectos, en un ámbito específico.

Tanta importancia adquiere esta dimensión que ha dado pie a la época del "proyectismo", como instrumento único de cooperación, que llegará a desplazar a las otras dimensiones de la cooperación. El interés por la gestión se centra en las cuestiones gerenciales con la necesaria preocupación por la eficacia y la eficiencia, y por la captación de recursos. Cooperar acaba siendo realizar un proyecto con subvención pública, sometido a los criterios de gestión administrativa, con sus burocracias y corporaciones.

Como consecuencia se producen dos efectos contraproducentes. En primer lugar se declara al "proyecto" como instrumento único de la cooperación y se debilitan tanto el fortalecimiento de las convicciones personal y colectivas como su condición de movimiento social; en segundo lugar, se convierte a los ejecutores de los proyectos en los actores fundamentales de la cooperación, renunciando a involucrar en el mundo de la cooperación a otros agentes sociales que pudieran aportar sus conocimientos y formular propuestas políticas más amplias que las que atañen solamente a la gestión estricta de los programas. El propio éxito de las ONGD se convertiría en su propio fracaso, que hace muy incierto su futuro. A partir de la crisis de los Grandes Lagos, donde las ONGD asumen tareas que no les correspon- 
den y que les exceden, se ha generado una creciente desconfianza en sus habilidades para resolver los problemas políticos y sociales.

\section{Otro modo de cooperar}

Caminamos en consecuencia hacia otro modo de ejercer la cooperación al desarrollo, con especial incidencia en la educación para el desarrollo, en la dimensión política de la cooperación, en el modo de ser una organización solidaria y en el potencial migratorio del codesarrollo.

Otro modo de ser estado en la esfera de la cooperación

La producción política de la cooperación necesita, ante todo, recrear las prácticas cooperativas y conectivas que fortalezcan la colaboración en lugar de las relaciones de dominio, y

\section{En las políticas de cooperación ne- cesitamos menos de la geopolítica y} más de ética de la indignación incapaz de consentir con el empobrecimiento; necesitamos menos del poder de los donantes, que de la cooperación humilde de los afectados.

el control desde la distancia se sustituya por dinámicas participativas con capacidad de promover otras relaciones. Ulrich BECK ha hablado de abajamiento y des-potenciamiento para abordar los nuevos problemas y riesgos. La cooperación al desarrollo propone otro modo de acción política "que tiende a movilizar todos los sectores de la sociedad" mediante una intervención directa que configure la cooperación desde abajo".

Se abandona, de este modo, la antitesis amigo-enemigo en el ámbito de la cooperación, que ha estado en la base de las corrupciones y amiguismo tan frecuentes en ciertas prácticas de la cooperación, y se abandonan igualmente los métodos burocráticos para generar nuevas prácticas mediante nuevas formas de organización, que germinalmente se están produciendo en organizaciones trasnacionales del tipo de Grenpace, Aministía internacional, Tierra de los Hombres, Foros sociales. En ellos aparecen los primeros síntomas de una ciudadanía mundial.

A nivel internacional, la Declaración del Milenio fue la victoria de la política sobre la economía, cuando la humanidad se propuso erradicar la pobreza hasta el año 2015. Era el triunfo de la capacidad de soñar, traída por la política: podemos erradicar la pobreza, si queremos; podemos crear un nuevo orden mundial, si pretendemos; podemos universalizar los derechos humanos, si codiciamos. Sin embargo, muy pronto se impuso una cierta economía sobre la política y, como consecuencia, se rebajaron todas las metas hasta convertir la erradicación de la pobreza (dominio de la política) en mera superación del hambre (dominio de la economía).

La fuerza de la cooperación no está tanto en la disponibilidad de medios fuertes y potentes, cuanto en la participación de la gente. Hay un uso de los medios fuertes y potentes en la cooperación que causa mayores males, cuando se hace sin comunidad, sin participación, sin colaboración.

En las políticas de cooperación necesitamos menos de la geopolítica, y más de ética de la indignación incapaz de consentir con el empobrecimiento; necesitamos menos del poder de los donantes, que de la cooperación humilde de los afectados, con capacidad de instituir relaciones, co-responsabilizar en las tareas y fomentar la compañía.

La mayor tarea que pueden acometer hoy las Administraciones pro-cooperación, consiste en reconocerse humildemente como un agente más y renunciar a la centralidad para entenderse como un simple facilitador. No se necesita la retirada del Estado de la esfera de la cooperación, ni siquiera su adelgazamiento, como pro- 
ponen los neoliberales y conservadores, sino recrear sus competencias y ejercer como Estado de otra manera. Cuando el Estado se coloca en el centro de la cooperación, los restantes agentes se desplazan a posiciones subordinadas. La situación de la cooperación vive, entonces, una excesiva "satelización". Por la vía de las subvenciones, el Estado ha colonizado a las organizaciones solidarias; por la vía de las acreditaciones excesivamente reglamentaristas se ha impedido la necesaria creatividad social; por la vía de las titularidades se ha debilitado el surgimiento de nuevas iniciativas; por la vía de las declaraciones retóricas sobre el sistema público se ha disminuido la responsabilidad social.

\section{Otro modo de ser ONGD}

Tres tipos de actores adquieren una importancia decisiva cuando se renuncia a la centralidad del Estado: los ciudadanos que recrean su propia responsabilidad social, mediante las comunidades de sentido; las organizaciones solidarias, que promueven movimientos sociales a través de la acción colectiva; las empresas sociales, que producen proyectos de cooperación. No se trata de eliminar ni de fragilizar el compromiso social del Estado, si no lo contrario; tampoco de entronizar las ONGD con prerrogativas divinas, más bien lo contrario; se busca lograr un mayor nivel de relaciones sinérgicas y reciprocidades entre los nuevos actores de la cooperación.

Las ONGD no estarán centradas en la recaudación de fondos públicos y recuperarán su estatuto de movimiento social; dejarán de ser simples intermediarias financieras que transfieren recursos al Sur para pasar a convertirse en dinamizadoras sociales en el Norte; dejarán de ser funcionales a los procesos económicos y políticos que acrecientan la pobreza para significarse como agentes de transformación que abran oportunidades para ejercitar las capacidades y potencialidades de las personas.

Como movimiento social convertirán la cooperación en un proceso bi-direccional que

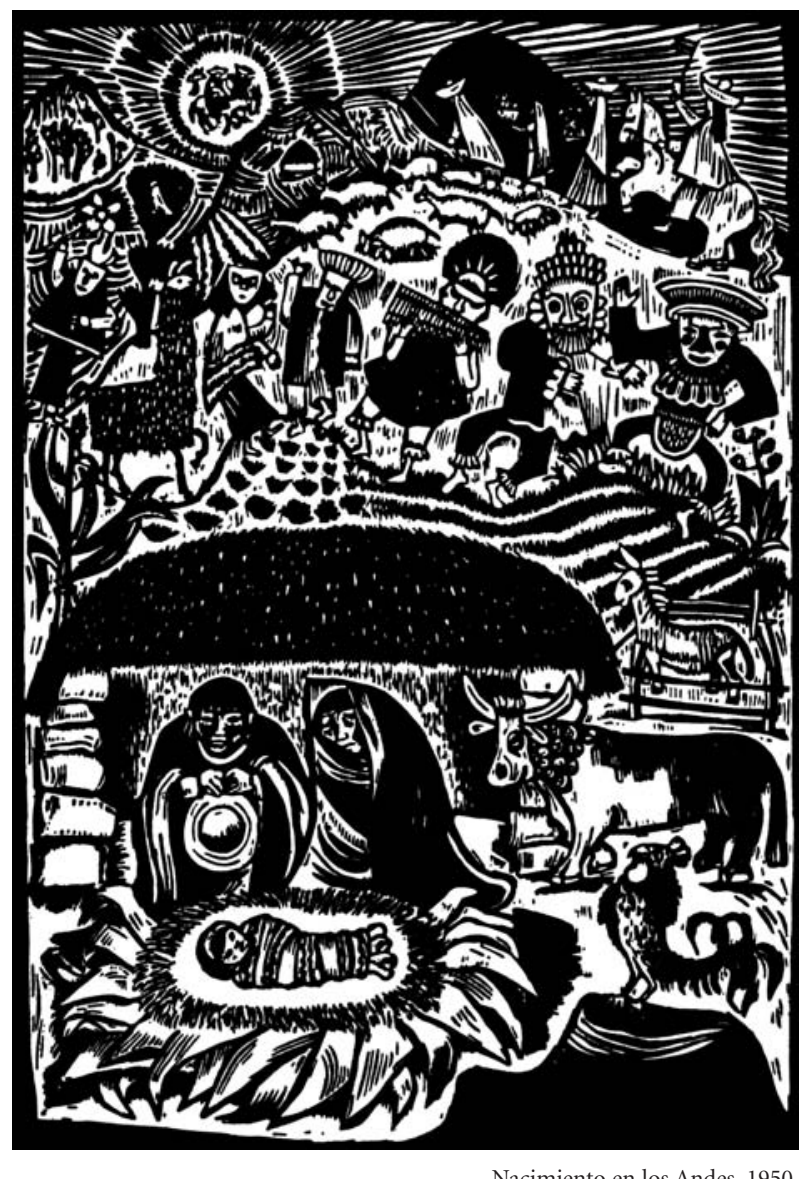

pone en cuestión el modelo de desarrollo de los países ricos. Las ONGD han de ceder el protagonismo a las propias comunidades y desarrollarán un simple papel de dinamizadores de intercambios, permitiendo así que la sociedad en su conjunto asuma la responsabilidad de la cooperación solidaria. Las Administraciones no instrumentalizarán a las ONGD en función de intereses económicos, políticos o culturales.

\section{Otro modo de gestión}

La actual administración de la cooperación ¿está en condiciones de gobernar las tres dimensiones de la cooperación con sus respectivos actores sociales? ¿Podrá afrontar la cooperación en condiciones de complejidad? En la actualidad, los departamentos gubernamentales de la cooperación están agrupados por sectores, en lugar de organizarse horizontalmente; de este modo, no 


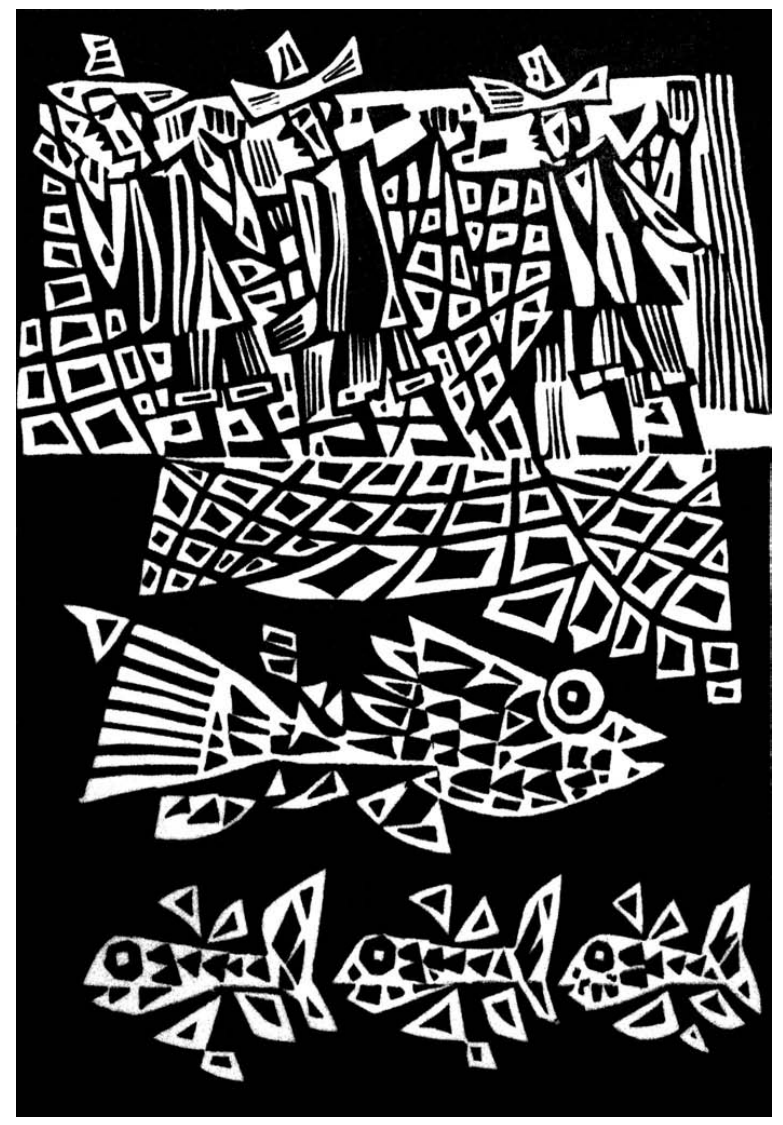

Ritual del río. 1983

están en condiciones para orientar procesos multidimensionales e integrados, realidades interdependientes y problemáticas interactivas. E incluso, son inadecuados para gobernar la multiplicidad de actores existentes e incorporarles activamente a la toma de decisiones.

En términos generales, la gobernación de la cooperación tiene dos alternativas ante sí: o bien se sigue como hasta ahora por la vía de agregar y sumar nuevos dispositivos a los ya existentes, o se plantea un salto cualitativo para gestionar los nuevos problemas. El primer itinerario propone aumentar los departamentos, agregar las prestaciones y crecer en unidades especializadas. Con frecuencia, las políticas pro-cooperación son en la práctica la suma de políticas sectoriales, no siempre armonizadas y a menudo contradictorias con conflictos intersectoriales y retraso en la toma de decisiones.
La segunda vía presta atención a las interacciones de los problemas y a las situaciones esencialmente mutantes; en lugar de un crecimiento por ampliación de lo existente, introduce mecanismos integradores, instituciones flexibles y participación ciudadana. La estabilidad de las instituciones, que era un objetivo básico del primer itinerario, no se contrapone a la elasticidad y prontitud para responder a necesidades y oportunidades en rápido cambio. Esta segunda vía requiere una revisión completa del sistema pro-cooperación a través de mejores mecanismos para la integración de políticas sectoriales e intercambios entre las administraciones y los actores sociales.

La tarea más urgente de los gestores públicos de la cooperación consiste en impulsar la doble transición -diferenciar para unir-, con objeto de fortalecer las relaciones entre las comunidades de sentido, que cultivan el valor de la cooperación, los movimientos sociales con incidencia socio-política y las organizaciones solidarias, que gestionan los proyectos. La responsabilidad pública enfatizará, de este modo, la lucha contra la pobreza mejorando los métodos de diálogo y colaboración.

\section{Migraciones y codesarrollo}

Las actuales migraciones hacen un aporte sustancial a la refundación de la cooperación al desarrollo. En cada inmigrante, cobran carne y sangre culturas, civilizaciones y religiones, que amplían las capacidades de todos para una vida más digna, justa y feliz. Detrás de cada inmigrante hay sueños que necesitamos todos para vivir humanamente; hay sueños de dignidad personal $\mathrm{y}$ aspiraciones a mejores condiciones de vida.

Cuando alguien sale de su país, con él viajan también sus redes sociales; la inmigración no es una aventura individual, sino que es un nuevo modo de vivir la vinculación con los suyos. El codesarrollo consiste en visibilizar esta vinculación a través de medidas concretas en las comunidades de origen, que permitan la utilización de las nuevas capacidades al servicio del desarrollo 
local, desde las remesas que promuevan estructuras financieras locales hasta las pequeñas cooperativas de ahorro y crédito para la dinamización de las pequeñas economías locales de los entornos migratorios, desde las capacidades culturales hasta los hábitos democráticos.

Las migraciones configuran vínculos, conexiones, redes y relaciones entre origen y destino, entre lugares distantes. El migrante es una conexión que mantiene vínculos con su tierra natal y de ella recibe noticias, peticiones, afectos, parientes. Este potencial de vinculación está generando campos sociales transnacionales: familias trasnacionales, política transnacional, escuelas transnacionales...

No cabe duda que la presencia de inmigrantes afecta positivamente a los países receptores y podrá resultar positiva a los países emisores a condición de que se active el codesarrollo, como forma de cooperación.

Resulta beneficiosa para la demografía de los países de acogida, ya que les ayudan a salir del estancamiento demográfico. Recientemente, la ONU, en su Informe de Desarrollo Humano, recomienda a Europa duplicar su número de inmigrantes hasta el año 2050 para compensar el envejecimiento de su población y la baja natalidad. Asimismo, ha producido un incremento de la población escolarizada y las cotizaciones a la Seguridad Social de la inmigración han hecho posible, en buena parte, el superávit anual que registra las cuentas de este organismo en los países del Norte. Puede decirse que el desarrollo del Norte sólo será sostenible si incorpora a personas inmigrantes.

Las migraciones traen efectos positivos, también, para sus países de origen: sostienen a las familias que allá quedaron; envían remesas que disminuyen las tensiones sociolaborales, aportan estilos de vida democratizadores, fortalecen los contactos entres las redes formales e informales, financian micro-proyectos, contribuyen a la transición demográfica de sus países. Pero, sobre todo, se están convirtiendo en un nuevo actor para la cooperación al desarrollo. El co- desarrollo es un modo de favorecer la participación activa de las personas migrantes en el compromiso por el desarrollo de sus comunidades de origen. Los inmigrantes son potenciales que pueden y deben ocuparse de sus países de origen. El codesarrollo enfatiza las capacidades de los propios inmigrantes como agentes de cooperación. Los inmigrantes tienen un papel importante en la creación de enlaces y conexiones entre países de origen y de acogida

Es asimismo un modo de entender la relación entre los países del Norte y los del Sur basada en la colaboración, reciprocidad y justicia. En el origen de las migraciones existe la ausencia de condiciones de vida y un reparto injusto y desigual del crecimiento económico, que se constituye así en el principal factor de expulsión de las migraciones forzosas.

Necesitamos abrir la cooperación al codesarrollo. Entendiendo que el co-desarrollo no es una medida destinada a invertir recursos en los países de origen de los inmigrantes con el fin de impedir que vengan. Tampoco se deben ligar las medidas de codesarrollo a las políticas de control migratorio. Ni siquiera es una medida destinada a estimular los retornos de los inmigrantes a sus pases mediante apoyos económicos: no se debe ligar las iniciativas de codesarrollo al retorno de los inmigrantes a su país de origen. Tampoco es una estrategia destinada a utilizar las remesas para el desarrollo de sus comunidades de origen como un ámbito de la actividad bancaria. Es un modo de fomentar la asociación entre los propios inmigrantes con el fin de establecer el diálogo y la presión tanto en sus países de origen como los de destino.

Con frecuencia, la inmigración se considera una aventura individual, por la cual alguien intenta solucionar su vida de modo individual. El codesarrollo, por el contrario, favorece la búsqueda de respuestas colectivas y asociadas.

De este modo, nace una nueva concepción de las políticas migratorias, que postula pasar del concepto de extranjería -la inmigración comienza en nuestra frontera- al de inmigración, que 
comienza cuando alguien debe abandonar el país de origen en busca de un mejor futuro y vida digna. La pregunta ya no consiste en saber qué se puede hacer cuando la persona llega a la frontera (extranjería), sino en aproximarse a un proceso que empieza en la salida y afecta a todos los momentos del trayecto (migración). ¿Por qué se sale? ¿Qué sucede en el camino? ¿Cómo se entra en el país receptor? ¿Qué cambios se producen en la identidad de la persona que emigra? ¿Cómo se trasforma la sociedad receptora en contacto con los inmigrantes? Si hay derecho a emigrar, existe también el derecho al retorno, basado en la libertad personal y en el compromiso a favor de un país que se ha visto empobrecido por la salida de ciudadanos llenos de coraje y competencia.

1 HABERMAS, J. El discurso filosófico de la modernidad, Madrid, Taurus, 1989.

2 RICOEUR, P. Du texte à l'action, Esprit, Seuil, 1986.

3 BENJAMIN, W. Tesis de filosofía de la historia, en Discursos interrumpidos. Madrid, Taurus, 1973.

4 HORKHEIMER, M. El espacio social, en Ocaso, Barcelona, Antrhopos, 1986, p.108.

4 BECK, U. Un nuevo mundo feliz, Barcelona, Paidós, 2000, pp. 25-26.

6 BOFF, L. Teología del cautiverio y de la liberación. Ed. Paulinas, Madrid, 1978,

7 GARCÍA ROCA, Globalización y solidaridad en OSORIO, ELIZALDE Ampliando El Arcoiris. Nuevos paradigmas en educación, política y desarrollo, Universidad Bolivariana, Santiago de Chile, 2005, pp. 92.

8 JONAS, H. El principio Vida. Hacia una biología filosófica, Trotta, Madrid, 2000.

9 BOFF, L. Ecología grito de la Tierra, grito de los pobres, Trotta, Madrid, 2002.

10 RITZER, G. The Globalization of Nothing, Pine Forge Press, California, 2004, BECK, U. Qué es la globalización. Falacias del globalismo, respuestas a la globalización, Paidós, Barcelona, 1998.

11 RIFKIN, J. El sueño europeo, Paidós, 2004.

12 NAIR, S. Y vendrán. Las migraciones en tiempos hostiles, Bronce, Barcelona, 2006.

13 BARBER, B. La interdependencia, en El País 10-9-04.

14 DELORS, J. La educación encierra un tesoro, Santillana, UNESCO, Madrid, 1996.

15 GARCÍA ROCA, J. Migracions i globalització, en L Espil 21 (2006)l

16 GARCIA ROCA, J. Globalización, en 10 palabras clave en filosofía política (coord.,Adela CORTINA), Estella: EVD, 1998, pp. 163-212.

17 HIRSCHMAN, A. Retóricas de la intransigencia, FCE, México, 1991.

18 ENSENSBERGER, H. M. Perspectivas de guerra civil, Barcelona, Anagrama, 1994.

19 ROBINSON, M. Los derechos humanos, ensombrecidos por el 11-S. en El PAÍS 4-7- 2002.

20 Sólo desde 1988 a 1996, los gastos anuales para la ayuda humanitaria por parte de los miembros de la OCDE han crecido de 410 millones a 3.066 millones de euros. La asistencia humanitaria de la Unión Europea para situaciones de emergencia ha pasado de 195.3 millones de euros en 1991 a 700 en 1997. Mientras que los fondos para la cooperación al desarrollo han disminuido un 25\%. RUFFINI, G. Il ruolo delle organización non governative nell'emergenza, en Movimondo. Dopo la Guerra, Roma, 1999.

21 Nigrizia, dossier Aiuto n. 7-8- 2000.

22 POMFRET .J. A chi vanno gli aiuti umanitari? In Internazionale (1997) 206, p. 7.

23 FALK, R. Resisting"Globalization from above" through "Globalization from below", New Political Economy 2 (1997), pp.17-24.

24 METZ, J. B. Jahrbuch Politische Theologie, vol 2. Bilderverbot, a cura de RAINER, M. Y JANSSEN, H-G. Münster, 1997. 